\title{
La flauta de pico en la escuela: la opinión del maestro de música
}

The recorder at school: the opinion of the music teacher

\author{
Josep Gustems-Carnicer \\ igustems@ub.edu \\ Departamento de Didácticas Aplicadas \\ Universidad de Barcelona \\ Barcelona, España \\ ORCID: http://orcid.org/0000-0002-6442-9805
}

Diego Calderón-Garrido

dcalderon@ub.edu

Departamento de Didácticas Aplicadas Serra Húnter Fellow, Universidad de Barcelona

Barcelona, España

ORCID: http://orcid.org/0000-0002-2860-6747

\author{
Mercè Navarro \\ mercenavarro55@hotmail.com \\ CEIP Turó de Can Mates \\ Barcelona, España \\ ORCID: http://orcid.org/0000-0002-4370-5479
}

Gloria Segura

gsespiell@gmail.com

Centro de Educación Musical Euterpiades

Barcelona, España

doi: 10.7203/LEEME.46.17260

Recibido: 30-04-20 Aceptado: 23-05-20. Contacto y correspondencia: Josep Gustems-Carnicer. Departamento de Didácticas Aplicadas, Universidad de Barcelona, Pg. Vall d'Hebron, 171, C.P. 08035 Barcelona. España.

\begin{abstract}
Resumen
La flauta de pico es un instrumento habitual en la escuela Primaria desde hace ya varias décadas. En este artículo, a través de un cuestionario on line ad hoc contestado por 605 docentes, se ha analizado su uso en la escuela, así como sus ventajas e inconvenientes, además de mostrar las propuestas de mejora realizadas por el profesorado. Los resultados muestran que se percibe como un instrumento fácil de aprender y de usar, para el que la mayoría de los docentes se sienten capacitados para enseñar a pesar de su poca formación específica, aunque tiene algunas limitaciones centradas mayoritariamente en la idiosincrasia del propio instrumento. Además, los maestros reconocen el potencial motivacional de la flauta si se sabe adecuar a los aspectos contextuales, edades y niveles. Destacaron que resulta un precursor muy valioso a la hora de aprender otro tipo de instrumentos. También sobresalieron tanto aspectos de entrenamiento auditivo, técnica, interpretación o improvisación, como de autocontrol, práctica, trabajo en equipo, compromiso y flexibilidad.
\end{abstract}

Palabras clave: Flauta de pico; educación musical; Educación Primaria; formación de maestros.

\section{Abstract}

The recorder has been a common instrument in Primary schools for several decades. In this article, through an ad hoc online questionnaire answered by 605 teachers, we have analyzed its use in schools, its advantages and disadvantages, and its influence on proposals for improvement made by teachers. The results show that recorder is perceived as an instrument that is easy to learn and use, for which most teachers feel qualified to teach in spite of their having little specific training and the fact that it has some limitations that are mostly centered on the idiosyncrasy of the instrument itself. In addition, teachers recognize the motivational potential of the instrument if it is adapted to the contextual aspects, ages, and levels. In addition, they stressed that it is a valuable precursor when it comes to learning other types of instruments. They also highlighted aspects of auditory training, technique, performance, or improvisation as well as self-control, practice, teamwork, commitment, and flexibility.

Key words: Recorder; music education; Primary Education; teacher training.

@ Josep Gustems-Carnicer, Diego Calderón-Garrido, Mercè Navarro y Gloria Segura. The content of this article is the sole responsibility of the authors. The Revista Electrónica de LEEME and Universitat de València are not liable for any legal actions that may arise involving the article's content. Revista Electrónica de LEEME - Lista Electrónica Europea de Música en la Educación-. http://ojs.uv.es/index/php/LEEME/index ISSN: 1575-9563. Editores: Universidad de Valencia y Jesús Tejada. Visibilidad de esta revista: SCOPUS, Emerging Sources Citation Index (Clarivate), EBSCO, CINDOC (CSIC), Citefactor, COPAC, Dialnet, DICE (CSIC), DOAJ, e-revistas (CSIC), EBSCO Premier, ERIH+, Gale Cengage Learning, IN-RECS, IRESIE, LATINDEX, MIAR, OCLC Worldcat, RESH, REDIB, RILM Core Journals, SUDOC, ULRICHS. Esta revista es de acceso libre mediante licencia Creative Commons $4.0 \mathrm{CC}$ by. Política de archivado: etiqueta verde SHERPA-ROMEO. 


\section{La flauta de pico, un instrumento con vocación universal}

Si existe un instrumento musical que ha sido asociado durante décadas a la escuela, este ha sido la flauta de pico o flauta dulce. Se trata de un instrumento modesto, aunque con una historia interesante y con muchas más prestaciones de las que se suele sacar partido en unas pocas clases colectivas. Al igual que ocurre con otros recursos didácticos, cabe plantearse si continúa vigente actualmente en la escuela y si los docentes y centros educativos apuestan por ella para abordar los retos que plantea la educación en este siglo.

Con el nombre de flauta de pico ( $\mathrm{F}$ desde aquí) se designa a un prototipo de instrumento presente a lo largo de la geografía mundial en todas las épocas y culturas. De hecho, es hasta la fecha el resto arqueológico musical más antiguo descubierto, datado con 42.000 años de antigüedad (Fitch, 2005; Lafarga y Sanz, 2014). A pesar de haber sido un instrumento muy relevante en Europa en la Edad Media, el Renacimiento y el Barroco, con un marcado sesgo mediterráneo y catalano-aragonés (Paquier, Hendrickx y Jeannin, 2016), la F perdió presencia con la preferencia por los grandes conjuntos sinfónicos en el clasicismo y romanticismo, que perseguían una expresividad vinculada, entre otros, a los cambios de intensidad. No obstante, la $\mathrm{F}$ pervivió de alguna manera a través de sus variantes populares, $\mathrm{F}$ de 6 agujeros, $\mathrm{F}$ para tocarse con una sola mano acompañada de percusión, ya sea de tres agujeros -tipo txistu- o con 5 como el flabiol. (Lubarsky, 2017; Robert, 1998).

El ansia de la musicología aplicada de fines del s. XIX por recrear escenarios y sonoridades reales de una época llevó a algunos investigadores a recuperar instrumentos antiguos en buen estado de conservación. Fue Inglaterra la impulsora de la "resurrección" de la F como instrumento histórico, así como de su repertorio, tratados y cursos de formación (Thomson, 1995). El impulso de restauración inglés se vio amplificado a nivel internacional por multitud de estudiosos que acudieron a sus cursos de verano y replicaron en sus países las experiencias estéticas vividas (Roudabush, 2017). Así, en 1939, se fundó la American Recorder Society en Inglaterra. Otros países como Suiza y Holanda se sumarían para profundizar en la música antigua que devendría un estandarte de pureza y veracidad interpretativas. Estas sociedades publicaron diferentes revistas científicas con afán divulgativo (como la American Recorder Magazine desde 1959, Recorder Education Journal en Reino Unido desde 1990, o The Recorder Magazine en Reino Unido, desde 1993) a las que se sumarían los catálogos editoriales de Moeck o Schott's. En España, apareció la revista Musica Antiqua en los años 80, la revista Flauta de Pico en los 90, así como la oferta de cursos de verano en La Seu d'Urgell, Canet, Daroca, o El Escorial, a partir del empuje recibido por la inclusión de la $\mathrm{F}$ en los conservatorios superiores, sobre todo los de Madrid y Barcelona (Escalas, 1972).

A pesar de esta acción reivindicativa y musicológica de los conservatorios y sociedades musicales, la gran popularidad de la F se debió a su inclusión en la educación musical escolar.

$@$ @osep Gustems-Carnicer, Diego Calderón-Garrido, Mercè Navarro y Gloria Segura. The content of this article is the sole responsibility of the authors. The Revista Electrónica de LEEME and Universitat de València are not liable for any legal actions that may arise involving the article's content. Revista Electrónica de LEEME - Lista Electrónica Europea de Música en la Educación-. http://ojs.uv.es/index/php/LEEME/index ISSN: 1575-9563. Editores: Universidad de Valencia y Jesús Tejada. Visibilidad de esta revista: SCOPUS, Emerging Sources Citation Index (Clarivate), EBSCO, CINDOC (CSIC), Citefactor, COPAC, Dialnet, DICE (CSIC), DOAJ, e-revistas (CSIC), EBSCO Premier, ERIH+, Gale Cengage Learning, IN-RECS, IRESIE, LATINDEX, MIAR, OCLC Worldcat, RESH, REDIB, RILM Core Journals, SUDOC, ULRICHS. Esta revista es de acceso libre mediante licencia Creative Commons $4.0 \mathrm{CC}$ by. Política de archivado: etiqueta verde SHERPA-ROMEO. 
Esto sucedió a partir de las propuestas de Orff y Keetman (1950) en Alemania, que se propagaron por toda Europa gracias a la construcción en serie de $\mathrm{F}$ de bajo precio, construidas de madera en un inicio y más tarde de plástico (Montoya, 2017). Algunos destacados pedagogos musicales de España como Cateura (1976) u Oriol (2004) se interesaron por el instrumento y lo incluyeron en sus propuestas escolares, así como en la formación inicial de maestros. En este sentido, la labor de editoriales como Alpuerto, Clivis, Artison o Rosa Sensat, entre otras, también facilitaron la expansión del instrumento en los diferentes ámbitos educativos españoles. La $\mathrm{F}$ vino a apoyar y complementar el canto, la audición o las actividades de movimiento presentes en las clases de música, pasando a ser la actividad preferida del alumnado (Bowles, 1999).

Desde entonces, la presencia de la $\mathrm{F}$ en la educación obligatoria ha sido una constante, buscando llegar a todo tipo de contextos escolares, procedencias e intereses y sacrificando en ocasiones su pureza estética para poder interpretar repertorios actuales más cercanos a instrumentos con otro tipo de recursos expresivos (Rodríguez y Álvarez, 2017). Los intentos por preservar la calidad, presentes en multitud de propuestas como las de Galofré (1980), Izquierdo, Jiménez y Montserrat (1996) o de la Associació de Flautistes BLOC (2015), han sido un contrapeso necesario a sistemas mucho más popularizadores, como las páginas web dedicadas al aprendizaje autónomo de la $\mathrm{F}^{1}$, cuyo primer catálogo de recursos se puede encontrar en el trabajo de Gustems y Cunill (2007). Así pues, su presencia en la formación de maestros y en el currículum escolar obligatorio es diversa y flexible, por lo que no existe homogeneidad ni una evaluación homologable de sus contenidos (Gustems, 2004).

La inclusión de la $\mathrm{F}$ en las escuelas estuvo también vinculada a algunas experiencias interesantes de agrupaciones musicales que justificaban la importancia del trabajo del conjunto instrumental en la Educación Primaria. Un claro ejemplo es el de José Antonio Abreu, fundador de la Orquesta Sinfónica Nacional y de la Fundación Estatal para el Sistema Nacional de la Juventud e Infantil de Venezuela (FESNOJIV). Para él, la música une a las personas a través de sus valores: compromiso, perseverancia, liderazgo, promoción de la autoestima, en definitiva, valores esenciales para la vida, para la sociedad y para favorecer la integración de todos los seres humanos (Gustems-Carnicer y Calderón-Garrido, 2016). A pesar de las claras diferencias entre el proyecto orquestal de Abreu y una agrupación de flautas, no cabe duda de que ambas experiencias musicales comparten muchos de los valores descritos. Según Ferrer (2009), una orquesta o conjunto instrumental debe atender a las instrucciones del director, el respeto por el resto del grupo, el hábito de escuchar y la atención, hábitos de disciplina y orden necesarios para lograr un buen resultado colectivo. En este escenario de desarrollo de valores, las escuelas han sabido beneficiarse incluyendo los conjuntos instrumentales propios de los conservatorios en las

${ }^{1}$ La más famosa en sus inicios, la Recorder Home Page de 1990 gestionada por Lander, Así como tutoriales, bases instrumentales, karaokes, etc.

@ Josep Gustems-Carnicer, Diego Calderón-Garrido, Mercè Navarro y Gloria Segura. The content of this article is the sole responsibility of the authors. The Revista Electrónica de LEEME and Universitat de València are not liable for any legal actions that may arise involving the article's content. Revista Electrónica de LEEME - Lista Electrónica Europea de Música en la Educación-. http://ojs.uv.es/index/php/LEEME/index ISSN: 1575-9563. Editores: Universidad de Valencia y Jesús Tejada. Visibilidad de esta revista: SCOPUS, Emerging Sources Citation Index (Clarivate), EBSCO, CINDOC (CSIC), Citefactor, COPAC, Dialnet, DICE (CSIC), DOAJ, e-revistas (CSIC), EBSCO Premier, ERIH+, Gale Cengage Learning, IN-RECS, IRESIE, LATINDEX, MIAR, OCLC Worldcat, RESH, REDIB, RILM Core Journals, SUDOC, ULRICHS. Esta revista es de acceso libre mediante licencia Creative Commons $4.0 \mathrm{CC}$ by. Política de archivado: etiqueta verde SHERPA-ROMEO. 
aulas de Primaria (Calderón-Garrido, 2014). Sin embargo, no se puede obviar que la selección de repertorios, tipos de conjuntos e incluso instrumentos están estrechamente influidos por la representación que tiene cada maestro sobre la propia música (Huillipan Rain y ÁngelAlvarado, 2020).

Con relación a la enseñanza de la $\mathrm{F}$ y su práctica en conjunto instrumental en la escuela, diversas experiencias docentes en el aula muestran dificultades a nivel musical, social y de aprendizajes (Jambrina, 2007). A nivel musical, el resultado sonoro de la $\mathrm{F}$ resulta algunas veces estridente y poco atractivo para los alumnos, sobre todo en sus inicios. A nivel social (familia, escuela, compañeros, etc.), la $\mathrm{F}$ es considerada un instrumento algo arcaico con el que el alumnado se siente poco identificado y tampoco socialmente reconocido, quien muestra preferencia por otros instrumentos de más actualidad como pueden ser la guitarra, el ukelele o teclado. En el ámbito de su aprendizaje, tampoco les resulta fácil adquirir correctamente su técnica (digitación, respiración y emisión) con el fin de obtener una afinación adecuada, aunque algunas experiencias sobre su aprendizaje en condiciones de intensificación han dado excelentes resultados (Egger, Springer y Gooding, 2013). En este sentido, sin duda, se trata de un instrumento con el que "el profesorado tendría dificultades para articular y equilibrar los fines musicales con los pedagógicos" (Huillipan Rain y Ángel-Alvarado, 2020, p.54).

Por todo ello, y a pesar del impulso comentado antes, actualmente se observa cierto estancamiento de su presencia en las escuelas y se echa en falta más investigaciones específicas en este campo. Esto lleva a indagar cuáles son sus causas y cómo reconducir esta enseñanza que parece de gran interés, a pesar de algunos problemas que se han comentado.

Así pues, el objetivo de esta investigación es conocer las percepciones y uso que hace el profesorado de la $\mathrm{F}$ en Educación Primaria a partir de las opiniones recogidas a una muestra de profesorado de música en ejercicio, de forma que se pueda reflexionar sobre su funcionalidad y vigencia en la educación actual. Se incluyen, además, algunas propuestas de mejora. Con todo ello, se pretende aportar ideas a la tarea docente del profesorado de música en ejercicio, así como a su formación inicial.

\section{Método}

\subsection{Diseño}

Para la consecución de este objetivo, se ha realizado un estudio basado en encuesta descriptivo, de tipo exploratorio, correlacional y transversal (Bisquerra, 2004) a través de la elaboración y aplicación de un cuestionario on line ad hoc. La elección de un cuestionario on line estuvo motivada por su capacidad para conseguir llegar a una gran muestra potencial. Sin

@Josep Gustems-Carnicer, Diego Calderón-Garrido, Mercè Navarro y Gloria Segura. The content of this article is the sole responsibility of the authors. The Revista Electrónica de LEEME and Universitat de València are not liable for any legal actions that may arise involving the article's content. Revista Electrónica de LEEME - Lista Electrónica Europea de Música en la Educación-. http://ojs.uv.es/index/php/LEEME/index ISSN: 1575-9563. Editores: Universidad de Valencia y Jesús Tejada. Visibilidad de esta revista: SCOPUS, Emerging Sources Citation Index (Clarivate), EBSCO, CINDOC (CSIC), Citefactor, COPAC, Dialnet, DICE (CSIC), DOAJ, e-revistas (CSIC), EBSCO Premier, ERIH+, Gale Cengage Learning, IN-RECS, IRESIE, LATINDEX, MIAR, OCLC Worldcat, RESH, REDIB, RILM Core Journals, SUDOC, ULRICHS. Esta revista es de acceso libre mediante licencia Creative Commons $4.0 \mathrm{CC}$ by. Política de archivado: etiqueta verde SHERPA-ROMEO. 
embargo, esto obviamente implica cierta subjetividad basada en la opinión o criterio del propio encuestado.

\subsection{Muestra}

Para la aplicación del cuestionario, se optó por la plataforma Google Forms. La muestra potencial estuvo compuesta por todos los profesores de música de escuelas públicas y privadas de Catalunya, unos 2.500 docentes. Se accedió a la muestra a través de las redes sociales y los correos personales. Se obtuvieron 623 respuestas, de las cuales se descartaron las que no habían completado el cuestionario en su totalidad. Finalmente se obtuvo una muestra válida de 605 participantes, de los cuales el $75.4 \%(n=456)$ fueron mujeres y el $24.6 \%(n=149)$ hombres. Sus edades se situaron mayoritariamente entre los 31 y 40 años $(\mathrm{n}=236 ; 39 \%)$, seguido del grupo de 41 a 50 años $(n=184 ; 30.4 \%)$.

Respecto a sus estudios, el $72.6 \%$ eran maestros con especialidad de Educación Musical, el 50.4\% tenían el Grado Profesional de Música, y el 22.6\% el Grado Superior. Respecto al tipo de centro, el $84 \%$ trabajaban en un centro público, el $15.7 \%$ en uno privadoconcertado y el $0.3 \%$ en uno privado. La mayoría de estos centros tenían dos líneas (44.5\%) o solo una (30.1\%); la gran mayoría de los participantes (92.1\%) impartían solo Música.

\subsection{Instrumento}

Para la elaboración del cuestionario, se recurrió a un panel de cuatro expertos que combinaba investigadores en educación con maestros en activo. Dichos expertos recibieron una primera versión realizada por los autores de esta investigación, sobre la que hicieron matizaciones y modificaciones tanto de texto y escala, así como de formato y orden de las diferentes preguntas. Posteriormente, esta segunda versión se aplicó a 20 docentes en activo, a los que se pidió una segunda opinión sobre la relevancia de las preguntas, la claridad de las mismas y el tiempo empleado en su contestación. En este proceso se consiguió un índice Kappa de Cohen de 0.82. Con esta segunda versión, se diseñó el instrumento final.

Tal como se muestra en la Tabla 1, el cuestionario definitivo se dividió en una primera dimensión de items sociolaborales y referidas a la formación genérica, seguido de otra sobre la formación en $\mathrm{F}$ de las personas encuestadas y, finalmente, otra sobre el uso de la $\mathrm{F}$ en la educación. El cuestionario quedó conformado por 18 ítems distribuidos en preguntas dicotómicas cerradas, preguntas politómicas, preguntas con escala tipo Likert y varias preguntas abiertas.

@ Josep Gustems-Carnicer, Diego Calderón-Garrido, Mercè Navarro y Gloria Segura. The content of this article is the sole responsibility of the authors. The Revista Electrónica de LEEME and Universitat de València are not liable for any legal actions that may arise involving the article's content. Revista Electrónica de LEEME - Lista Electrónica Europea de Música en la Educación-. http://ojs.uv.es/index/php/LEEME/index ISSN: 1575-9563. Editores: Universidad de Valencia y Jesús Tejada. Visibilidad de esta revista: SCOPUS, Emerging Sources Citation Index (Clarivate), EBSCO, CINDOC (CSIC), Citefactor, COPAC, Dialnet, DICE (CSIC), DOAJ, e-revistas (CSIC), EBSCO Premier, ERIH+, Gale Cengage Learning, IN-RECS, IRESIE, LATINDEX, MIAR, OCLC Worldcat, RESH, REDIB, RILM Core Journals, SUDOC, ULRICHS. Esta revista es de acceso libre mediante licencia Creative Commons $4.0 \mathrm{CC}$ by. Política de archivado: etiqueta verde SHERPA-ROMEO. 
Tabla 1. Dimensiones y preguntas del cuestionario administrado

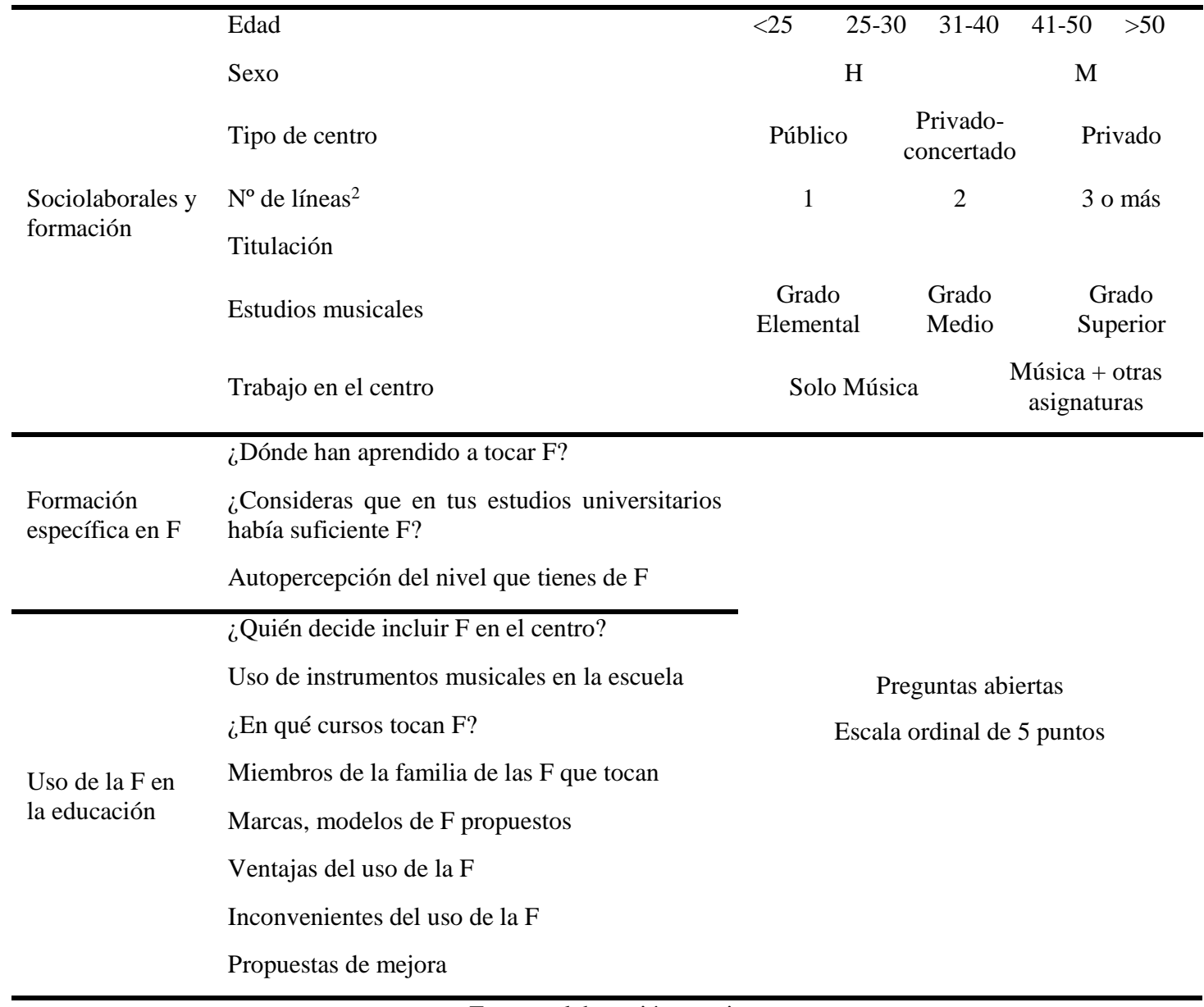

Fuente: elaboración propia

\subsection{Procedimiento}

En el propio cuestionario, se incluyó un consentimiento libre, previo e informado según el cual cada participante podía abandonar el estudio en el momento que desease. Al docente se le informaba que la cumplimentación del cuestionario le tomaría unos 10 minutos y se concedió 21 días para completarlo.

Además de los estadísticos descriptivos, y al ser datos no paramétricos, se buscaron las diferencias estadísticas con las pruebas de Kruskal-Wallis y de Mann-Whitney (Martínez, Castellanos y Chacón, 2014), en función de los diversos parámetros indicados en el cuestionario usado. Para el recuento y análisis estadístico de los resultados, se utilizó el programa Statistic

\footnotetext{
${ }^{2}$ El número de líneas hace referencia al número de clases o aulas diferentes en el mismo curso académico.

@ Josep Gustems-Carnicer, Diego Calderón-Garrido, Mercè Navarro y Gloria Segura. The content of this article is the sole responsibility of the authors. The Revista Electrónica de LEEME and Universitat de València are not liable for any legal actions that may arise involving the article's content. Revista Electrónica de LEEME - Lista Electrónica Europea de Música en la Educación-. http://ojs.uv.es/index/php/LEEME/index ISSN: 1575-9563. Editores: Universidad de Valencia y Jesús Tejada. Visibilidad de esta revista: SCOPUS, Emerging Sources Citation Index (Clarivate), EBSCO, CINDOC (CSIC), Citefactor, COPAC, Dialnet, DICE (CSIC), DOAJ, e-revistas (CSIC), EBSCO Premier, ERIH+, Gale Cengage Learning, IN-RECS, IRESIE, LATINDEX, MIAR, OCLC Worldcat, RESH, REDIB, RILM Core Journals, SUDOC, ULRICHS. Esta revista es de acceso libre mediante licencia Creative Commons $4.0 \mathrm{CC}$ by. Política de archivado: etiqueta verde SHERPA-ROMEO.
} 
Package for Social Science (SPSS) v21.0 y NVIVO para el análisis de texto de las respuestas abiertas.

\section{Resultados y discusión}

La primera pregunta es "Dónde ha aprendido a tocar la F". Las respuestas de los participantes se refirieron a su propia escolaridad o a los estudios de maestro (36.9\%), de forma autodidacta (20.6\%) o ampliándolos con estudios no formales (17.4\%). La $\mathrm{F}$ se percibe habitualmente como un instrumento sencillo para el profesorado de las escuelas. En este sentido, el autodidactismo es muy importante en este ámbito y coincide con la percepción que tenía el profesorado universitario de formación de maestros respecto su propia formación (Gustems, 2004).

Respecto a los contenidos de $\mathrm{F}$ en los estudios universitarios (formación inicial), la mayoría destacaron su poca (34.7\%) o escasa presencia (29.8\%). Si bien puede entenderse la formación en $\mathrm{F}$ en un contexto más amplio y como parte de un currículo formativo que comprende varios aspectos. Este dato constata una demanda generalizada sobre la escasa formación disciplinar que existe actualmente en la formación inicial de maestros (Chao, Mato y López, 2015), la cual se reduce a la Mención de Música, limitándose en torno a 30 créditos lectivos o 4-6 asignaturas en función de la Universidad en la que se realice. Obviamente, no siempre existe una relación directa entre el número de créditos y la formación en $\mathrm{F}$, pero se puede establecer que, a mayor espacio formativo, más posibilidades de que dicha formación comprenda entre otros aspectos el uso de la F. Por todo ello, puede plantearse si la formación en F del profesor de Educación Musical es suficiente para asegurar un correcto nivel competencial con el que impartir las asignaturas relacionadas con la educación musical.

Según su propia percepción sobre su nivel de $\mathrm{F}$, la mayoría de las personas encuestadas creía que era bueno, calificándolo como bastante $(n=267 ; 44.1 \%)$, o mucho $(n=224 ; 37 \%)$, lo que contrastaba con la escasa formación en $\mathrm{F}$ efectivamente recibida en los estudios universitarios. Estos datos estaban condicionados por el nivel de estudios musicales, mostrando diferencias significativas entre estas variables $\left(X^{2}{ }_{4}=18.018 ; p<0.001\right)$. De esta forma y tal como se puede observar en la Tabla 2, puntuaron más alto aquellos con estudios de Grado Superior e incluso Profesional (dicho dato se ha resaltado en la Tabla 2), aunque no sean específicamente en F. Esto podría hacer pensar que una enseñanza musical de nivel superior podría dotar de competencias técnicas y artísticas más allá del instrumento principal de la titulación, habilitando para mostrar capacidades de interpretación en otros contextos. Esta hipótesis confirmaría la intención de formación integral del músico perseguida por los Conservatorios Superiores (López de Arenosa, 2016). En la siguiente tabla, se muestra el número de respuestas en función de los estudios musicales y el nivel autopercibido de F,

$@$ @osep Gustems-Carnicer, Diego Calderón-Garrido, Mercè Navarro y Gloria Segura. The content of this article is the sole responsibility of the authors. The Revista Electrónica de LEEME and Universitat de València are not liable for any legal actions that may arise involving the article's content. Revista Electrónica de LEEME - Lista Electrónica Europea de Música en la Educación-. http://ojs.uv.es/index/php/LEEME/index ISSN: 1575-9563. Editores: Universidad de Valencia y Jesús Tejada. Visibilidad de esta revista: SCOPUS, Emerging Sources Citation Index (Clarivate), EBSCO, CINDOC (CSIC), Citefactor, COPAC, Dialnet, DICE (CSIC), DOAJ, e-revistas (CSIC), EBSCO Premier, ERIH+, Gale Cengage Learning, IN-RECS, IRESIE, LATINDEX, MIAR, OCLC Worldcat, RESH, REDIB, RILM Core Journals, SUDOC, ULRICHS. Esta revista es de acceso libre mediante licencia Creative Commons $4.0 \mathrm{CC}$ by. Política de archivado: etiqueta verde SHERPA-ROMEO. 
especificándose también qué porcentaje de respuestas correspondería a cada uno de dichos niveles autopercibidos.

Tabla 2. Autopercepción del nivel de flauta de los participantes según su nivel de estudios musicales

\begin{tabular}{lccccc} 
& Nada & Poco & Suficiente & Bastante & Mucho \\
\hline Ninguno & $3(8.6 \%)$ & $3(8.6 \%)$ & $8(22.9 \%)$ & $13(37.1 \%)$ & $8(22.9 \%)$ \\
No formales & & $1(5.3 \%)$ & $12(63.2 \%)$ & $6(31,6 \%)$ \\
Grado elemental & & $1(0.9 \%)$ & $18(16.5 \%)$ & $55(50.5 \%)$ & $35(32.1 \%)$ \\
Grado profesional & & $10(3.3 \%)$ & $49(16.1 \%)$ & $\mathbf{1 4 1}(\mathbf{4 6 . 2 \%})$ & $\mathbf{1 0 5}(\mathbf{3 4 . 3 \%})$ \\
Grado superior & $1(0.7 \%)$ & $6(4.4 \%)$ & $14(10.2 \%)$ & $46(33.6 \%)$ & $\mathbf{7 0}(\mathbf{5 0 . 1 \%})$ \\
\hline
\end{tabular}

Fuente: elaboración propia

Respecto a la decisión de usar la F en los centros, el 84.5\% de la muestra respondió que era el propio maestro quien lo decidía, solo o consensuadamente con la dirección. Solo el 10.9\% lo hacían por seguir con la costumbre del centro (Tabla 3).

Tabla 3. Toma de decisión del uso de la flauta en el aula de música

\begin{tabular}{lccc}
\cline { 2 - 3 } & Público & Concertado & Privado \\
\hline Ya estaba así & $51(10 \%)$ & $15(15.8 \%)$ & \\
Maestro/departamento de música & $\mathbf{3 7 6 ( 7 4 \% )}$ & $\mathbf{6 3}(\mathbf{6 3 . 2 \%})$ & $2(100 \%)$ \\
Maestro de música consensuada con el alumnado & $2(0.4 \%)$ & & \\
Maestro de música consensuada con dirección & $\mathbf{6 2 ( 1 2 . 2 \% )}$ & $11(11.6 \%)$ & \\
Dirección & $17(3.3 \%)$ & $9(9.5 \%)$ \\
\hline
\end{tabular}

Fuente: elaboración propia

Respecto al uso instrumentos musicales en al aula, destacó la $\mathrm{F}$, la percusión (sin especificar de qué tipo) y la voz. Además, el profesorado utilizaba bastante el piano y menos la guitarra (u otros instrumentos de la familia de tecla y cuerda pulsada, como el ukelele) para trabajar en el aula. El uso del flabiol, a pesar de las adaptaciones y propuestas para su uso escolar, seguía siendo muy limitado (Tabla 4).

@Josep Gustems-Carnicer, Diego Calderón-Garrido, Mercè Navarro y Gloria Segura. The content of this article is the sole responsibility of the authors. The Revista Electrónica de LEEME and Universitat de València are not liable for any legal actions that may arise involving the article's content. Revista Electrónica de LEEME - Lista Electrónica Europea de Música en la Educación-. http://ojs.uv.es/index/php/LEEME/index ISSN: 1575-9563. Editores: Universidad de Valencia y Jesús Tejada. Visibilidad de esta revista: SCOPUS, Emerging Sources Citation Index (Clarivate), EBSCO, CINDOC (CSIC), Citefactor, COPAC, Dialnet, DICE (CSIC), DOAJ, e-revistas (CSIC), EBSCO Premier, ERIH+, Gale Cengage Learning, IN-RECS, IRESIE, LATINDEX, MIAR, OCLC Worldcat, RESH, REDIB, RILM Core Journals, SUDOC, ULRICHS. Esta revista es de acceso libre mediante licencia Creative Commons $4.0 \mathrm{CC}$ by. Política de archivado: etiqueta verde SHERPA-ROMEO. 
Tabla 4. Uso de los instrumentos musicales en la escuela

\begin{tabular}{lccccccc} 
& Flauta & Flabiol & Percusión & Voz & Instrumentos Orff & Guitarra & Teclado \\
\hline No utilizan & 59 & $\mathbf{5 6 0}$ & 68 & 35 & 52 & $\mathbf{3 1 2}$ & 149 \\
Poco & 80 & 44 & 87 & 20 & 132 & 144 & 111 \\
Bastante & $\mathbf{2 7 2}$ & 1 & $\mathbf{2 5 6}$ & $\mathbf{1 1 8}$ & $\mathbf{2 4 4}$ & 92 & $\mathbf{2 0 8}$ \\
Mucho & $\mathbf{1 9 4}$ & & $\mathbf{1 9 4}$ & $\mathbf{4 3 2}$ & $\mathbf{1 7 7}$ & 57 & 137 \\
\hline \multicolumn{7}{c}{ Fuente: elaboración propia }
\end{tabular}

Fuente: elaboración propia

Respecto al uso de la F, se les preguntó en qué curso se introducía el instrumento. La mayoría de las respuestas la situaron en entre tercero y sexto de Primaria (70.9\%). Este dato coincide con la mayoría de las propuestas en libros de texto, como por ejemplo el de Galofré (1980) o el de Izquierdo, Jiménez y Montserrat (1996), pero contrasta con unos pocos que ya proponían hacerlo en todos los cursos de Primaria (Cateura, 1976).

Otro tema de interés era qué miembros de la familia de las $\mathrm{F}$ eran utilizados en la escuela. En este sentido, hubo diversidad de opiniones: mientras que 314 (51.9\%) introducían también otras F además de la soprano (contralto, tenor), muchos otros (47.1\%) solo trabajan con la F soprano.

A continuación, se les preguntó por el modelo de $\mathrm{F}$ que recomendarían. La mayoría (57.4\%) admitieron preferir los modelos de plástico frente a los de madera (8.6\%), aunque muchos no se decidían por ninguna o no contestaban (30.3\%). No hubo indicaciones ni diferencias respecto a la digitación barroca o alemana en este sentido.

Respecto a las ventajas del uso de la $\mathrm{F}$ en la escuela, hubo más respuestas referidas a sus ventajas que a sus inconvenientes (233 frente a 197). Así, por ejemplo, se manifestaba que "a pesar de las críticas de los adultos sobre sus recuerdos de cuando estudiaban flauta, en general, y según mi experiencia, a los alumnos les gusta ver que hacen música con un instrumento que suena enseguida" (Informante 255). Además, el análisis textual permitió agrupar las respuestas abiertas en cuatro grandes bloques (alumnado, profesorado, centro y familia), a partir de los nodos más relevantes que caracterizan dichas respuestas (Figura 1).

De esas opiniones favorables, destacaron las referidas al alumnado (52\%), en especial, la motivación que genera el trabajo en equipo, pues implica respeto, ayuda mutua y cooperación. Uno de los entrevistados lo resumía perfectamente: "la flauta es ideal para unificar al grupo" (Informante 592). Los contenidos cognitivos y emocionales vinculados con el aprendizaje de un instrumento (autoestima, esfuerzo, hábitos de estudio, atención...) quedan reforzados con la práctica instrumental, tal como lo evidencian multitud de investigaciones (García, del Olmo y Gutiérrez-Rivas, 2014; Kokotsaki y Hallam, 2013). Además, se señalaba

@Josep Gustems-Carnicer, Diego Calderón-Garrido, Mercè Navarro y Gloria Segura. The content of this article is the sole responsibility of the authors. The Revista Electrónica de LEEME and Universitat de València are not liable for any legal actions that may arise involving the article's content. Revista Electrónica de LEEME - Lista Electrónica Europea de Música en la Educación-. http://ojs.uv.es/index/php/LEEME/index ISSN: 1575-9563. Editores: Universidad de Valencia y Jesús Tejada. Visibilidad de esta revista: SCOPUS, Emerging Sources Citation Index (Clarivate), EBSCO, CINDOC (CSIC), Citefactor, COPAC, Dialnet, DICE (CSIC), DOAJ, e-revistas (CSIC), EBSCO Premier, ERIH+, Gale Cengage Learning, IN-RECS, IRESIE, LATINDEX, MIAR, OCLC Worldcat, RESH, REDIB, RILM Core Journals, SUDOC, ULRICHS. Esta revista es de acceso libre mediante licencia Creative Commons $4.0 \mathrm{CC}$ by. Política de archivado: etiqueta verde SHERPA-ROMEO. 
también el papel iniciador del instrumento, preparador para el posterior aprendizaje de algún otro instrumento.

Respecto a las ventajas para el profesorado y la asignatura de Música, un 31.3\% de las respuestas hacían referencia a que la $\mathrm{F}$ es una buena herramienta de aprendizaje musical, para iniciarse en un instrumento musical, para aplicar el lenguaje musical aprendido y para la práctica de conjunto instrumental. En este sentido, tal como se afirmó, "es un instrumento que permite acercarse de manera sencilla a los elementos del lenguaje musical" (Informante 315), además de que "permite formar conjuntos musicales completos usando también instrumental Orff mediano" (Informante 505). Respecto a las ventajas para las familias (13.3\%), la mayoría hacían referencia al bajo precio del instrumento, lo que la pondría al alcance de todos en pro de una democratización de la educación musical, especialmente desde que se fabrica en plástico (Hunt, 1978). Finalmente, destacó el uso de la $\mathrm{F}$ en conciertos escolares como principal ventaja para el centro, refiriéndose a beneficios para toda la institución, como son la socialización o la visibilización, ya que de dichos conciertos escolares se aprovechaban, además de los propios intérpretes, el resto de alumnado, profesorado e incluso familiares.

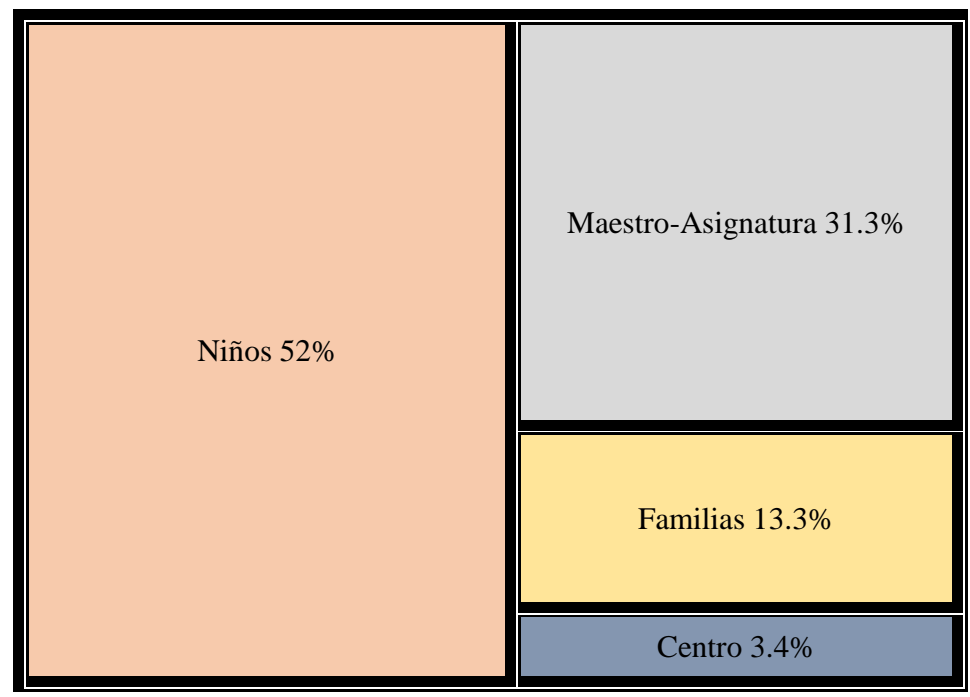

Figura 1. Ventajas del uso de la flauta, según quienes se benefician. Fuente: elaboración propia

Respecto a los inconvenientes del uso de la F en la escuela (Figura 2), la mayoría se referían al maestro y la asignatura de Música (55\%), destacando la falta de tiempo, trabajar con grupos demasiado numerosos y la dificultad de gestión del aula derivada de los diferentes niveles y tipología de alumnado, lo que resultaba estresante para los docentes. Tal es el caso de la siguiente afirmación:

Al tocar la flauta, se suman muchas habilidades: respiración, dicción, lectura rítmicomelódica, digitación, afinación, etc. Actualmente en la escuela tenemos una diversidad muy grande de alumnado, y los instrumentos Orff (xilófonos, metalófonos y carillones) me dan

@ Josep Gustems-Carnicer, Diego Calderón-Garrido, Mercè Navarro y Gloria Segura. The content of this article is the sole responsibility of the authors. The Revista Electrónica de LEEME and Universitat de València are not liable for any legal actions that may arise involving the article's content. Revista Electrónica de LEEME - Lista Electrónica Europea de Música en la Educación-. http://ojs.uv.es/index/php/LEEME/index ISSN: 1575-9563. Editores: Universidad de Valencia y Jesús Tejada. Visibilidad de esta revista: SCOPUS, Emerging Sources Citation Index (Clarivate), EBSCO, CINDOC (CSIC), Citefactor, COPAC, Dialnet, DICE (CSIC), DOAJ, e-revistas (CSIC), EBSCO Premier, ERIH+, Gale Cengage Learning, IN-RECS, IRESIE, LATINDEX, MIAR, OCLC Worldcat, RESH, REDIB, RILM Core Journals, SUDOC, ULRICHS. Esta revista es de acceso libre mediante licencia Creative Commons $4.0 \mathrm{CC}$ by. Política de archivado: etiqueta verde SHERPA-ROMEO. 
una diversidad más grande para poder adaptarme mejor al nivel de cada alumno (Informante 53).

Además, algunos docentes manifestaban las limitaciones generadas por el propio instrumento, como que "la flauta tiene muy poca variedad tímbrica" (Informante 4).

En relación a los inconvenientes referidos a los niños (33\%), algunos docentes comentaron de forma preferente que, en ocasiones, apreciaban una falta de motivación debida a un ocasional mal resultado sonoro colectivo y a las dificultades en tocar bien el instrumento. En este sentido, no se conformaban con un primer resultado sonoro, el cual se obtenía muy fácilmente, pero carente en muchas ocasiones de una calidad mínima. De esta forma, algunos de los inconvenientes no son exclusivos de las F, sino que podrían transferirse a otros ámbitos, como quien argumentaba que "les cuesta coger hábito de estudio"(Informante 2). Sin embargo, alguno de los docentes hacía referencia a la desigualdad de niveles, como por ejemplo que "los diferentes ritmos de aprendizaje son muy marcados. Los que hacen música fuera de la escuela pueden cansarse de ir a un ritmo demasiado lento y aquellos que, por naturaleza, les cuesta, se desmotivan porque ven que no llegarán a hacer lo que hacen sus compañeros" (Informante 71).

A continuación, se situarían los inconvenientes respecto al centro (8\%), entre los que destacaba el hecho de no ser un instrumento tradicional propio de su ámbito cultural y social que vincule al alumnado con su contexto y que, además, "está más relacionado con la escuela que con la música que escuchan los alumnos" (Informante 108). Finalmente, en algunos casos, y a pesar de que para otros docentes supuso una ventaja, los inconvenientes respecto a las familias (4\%) se refieren a su precio que, en ocasiones, puede resultar gravoso en algunos segmentos sociales ya que "a pesar de ser un instrumento económico, si queremos un mínimo de calidad, esta no está al alcance de todas las familias" (Informante 197).

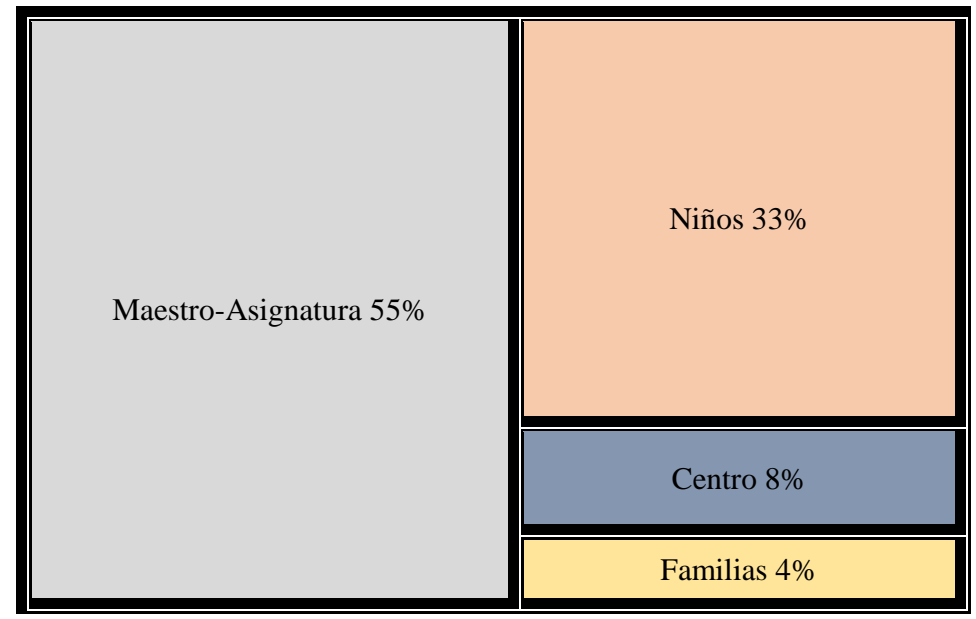

Figura 2. Inconvenientes del uso de la flauta, según a quienes perjudica. Fuente: elaboración propia

@ Josep Gustems-Carnicer, Diego Calderón-Garrido, Mercè Navarro y Gloria Segura. The content of this article is the sole responsibility of the authors. The Revista Electrónica de LEEME and Universitat de València are not liable for any legal actions that may arise involving the article's content. Revista Electrónica de LEEME - Lista Electrónica Europea de Música en la Educación-. http://ojs.uv.es/index/php/LEEME/index ISSN: 1575-9563. Editores: Universidad de Valencia y Jesús Tejada. Visibilidad de esta revista: SCOPUS, Emerging Sources Citation Index (Clarivate), EBSCO, CINDOC (CSIC), Citefactor, COPAC, Dialnet, DICE (CSIC), DOAJ, e-revistas (CSIC), EBSCO Premier, ERIH+, Gale Cengage Learning, IN-RECS, IRESIE, LATINDEX, MIAR, OCLC Worldcat, RESH, REDIB, RILM Core Journals, SUDOC, ULRICHS. Esta revista es de acceso libre mediante licencia Creative Commons $4.0 \mathrm{CC}$ by. Política de archivado: etiqueta verde SHERPA-ROMEO. 
Finalmente, se pedía a los participantes que hiciesen propuestas de mejora para el buen uso de la $\mathrm{F}$ en la escuela. La mayoría (54\%) se refería a utilizar de forma más generalizada un repertorio motivador, proponiendo la utilización de bases instrumentales grabadas desde diferentes páginas web. Así, por ejemplo, consideraban que "hay que cambiar el enfoque que se le da a la F. Muchas escuelas no la tocan por el miedo a caer en la rutina: mismas canciones, poco uso en un futuro, etc."(Informante 8). Otro informante afirmaba que "si las canciones son las adecuadas, los alumnos aprenden enseguida y hay muchos tutoriales en Youtube que apoyan la labor que hacemos en el aula, especialmente los acompañamientos" (Informante 128).

Además, también proponían que el estudio de la $\mathrm{F}$ fuese complementario al de otros instrumentos, como la voz, percusiones, instrumental Orff, teclados, ukelele; como es el caso, por ejemplo, de quien que considera que "la complementaría con la voz y el ukelele" (Informante 86). En último lugar, se señala la necesidad de asegurar un buen nivel de $\mathrm{F}$ por parte del profesorado, ya sea mediante formación inicial o permanente, como destaca quien piensa que "el problema es la poca formación que tienen muchos colegas, por lo que siempre recurren a lo mismo" (Informante 315).

El resto de las propuestas guardaba relación con decisiones organizativo-académicas de centro: ubicación dentro del programa curricular (la mayoría en $4^{\circ}, 5^{\circ}$ y $6^{\circ}$ cursos) y trabajo en desdobles o grupos reducidos, opcionales o voluntarios, como que afirmaba quien "considero muy importante trabajar desde los inicios el sonido en pequeños conjuntos para conseguir un sonido dulce y bien afinado"(Informante 114).

También, se mencionó la propuesta de utilizar el mismo modelo y marca de F para evitar algunos problemas de afinación."Es un instrumento que me gusta mucho y me gusta aprender y enseñar con los alumnos utilizándola. Utilizamos un modelo de flauta barroca y unificamos todos con la misma marca para descartar problemas con afinaciones diversas" (Informante 260).

\section{Conclusiones}

El objetivo de esta investigación ha sido profundizar en el uso de la $\mathrm{F}$ en la Educación Primaria, recogiendo sus principales formas de uso, así como sus ventajas e inconvenientes, junto con algunas propuestas de mejora. En este sentido, los resultados señalan que la $\mathrm{F}$ es un instrumento muy utilizado en las escuelas, a pesar de algunas limitaciones. En general, se percibe como un instrumento fácil de usar y aprender, para cuyo dominio se siente capacitado la mayor parte del profesorado, a pesar de que la mayoría haya manifestado su poca formación específica. Sin embargo, su uso generalizado se ve limitado por aspectos referidos al propio sonido del instrumento y, sobre todo, a aspectos organizativos del aula de música. Los referidos a la asignatura son de difícil solución en el contexto educativo actual, muy tensionado por los

$@$ @osep Gustems-Carnicer, Diego Calderón-Garrido, Mercè Navarro y Gloria Segura. The content of this article is the sole responsibility of the authors. The Revista Electrónica de LEEME and Universitat de València are not liable for any legal actions that may arise involving the article's content. Revista Electrónica de LEEME - Lista Electrónica Europea de Música en la Educación-. http://ojs.uv.es/index/php/LEEME/index ISSN: 1575-9563. Editores: Universidad de Valencia y Jesús Tejada. Visibilidad de esta revista: SCOPUS, Emerging Sources Citation Index (Clarivate), EBSCO, CINDOC (CSIC), Citefactor, COPAC, Dialnet, DICE (CSIC), DOAJ, e-revistas (CSIC), EBSCO Premier, ERIH+, Gale Cengage Learning, IN-RECS, IRESIE, LATINDEX, MIAR, OCLC Worldcat, RESH, REDIB, RILM Core Journals, SUDOC, ULRICHS. Esta revista es de acceso libre mediante licencia Creative Commons $4.0 \mathrm{CC}$ by. Política de archivado: etiqueta verde SHERPA-ROMEO. 
diferentes informes de rendimiento intelectual, como PISA, centrados únicamente en unas determinadas competencias, donde la Música tiene una incidencia muy limitada, lo que se ha traducido en un detrimento progresivo de la carga lectiva y el número de horas semanales de la asignatura.

Los inconvenientes de su uso atañen sobre todo al profesorado de Música y a sus dificultades de gestión del aula, lo que también lleva a una reflexión en el sentido de promover mejores prácticas en educación musical a partir de un esfuerzo organizativo que permita una ratio más conveniente para actividades que requieren de una gran atención y silencio (Tripiana, 2016).

Respecto a las ventajas, el profesorado participante es consciente del papel motivacional de este instrumento entre el alumnado, si se sabe adecuar a los aspectos contextuales, edades y niveles de estos. Asimismo, se propone gran cantidad de recursos que actualizan la metodología de trabajo en la escuela, dando paso a las TIC en el aula (Calderón-Garrido, Cisneros, García y de las Heras, 2019), combinando la audición de piezas creadas para el consumo musical masivo con la experiencia real de tocar un instrumento acústico. Entre sus ventajas, el profesorado ha aludido tanto a aspectos de entrenamiento auditivo, técnica, interpretación o improvisación, como de autocontrol, práctica, trabajo en equipo, compromiso o flexibilidad (Young, Burwell y Pickup, 2003). No obstante, la F debe combinarse de forma complementaria con la voz y otros instrumentos musicales que den la oportunidad a todo el alumnado a expresarse musicalmente. Los estudios de preferencias instrumentales, como el de Ben-Tovim y Boyd (1987), Wrape, Dittloff y Callahan (2014), e incluso el de Kang (2018), señalan un perfil característico del estudiante de F que no siempre coincide con todo el alumnado de la escuela. Por ello, no se debe centrar demasiado el trabajo musical a partir de la F si se quiere atender a la heterogeneidad del alumnado de los centros de Educación Primaria.

Una de las razones del estancamiento que se percibe está relacionada con el perfil del profesorado de educación musical, que ha cambiado en los últimos años, una nueva generación que opta por enseñar otros instrumentos más actuales y cercanos al alumnado (ukelele, teclado o guitarra) y que está estrechamente vinculada a las tecnologías digitales, utilizando programas y aplicaciones musicales que facilitan su aprendizaje. De esta forma, a su vez, las nuevas vías de aprendizaje ponen en sintonía el mundo extraescolar con el del aula, así como las técnicas necesarias para interpretar música más actual. En esta línea, se está desarrollando proyectos musicales relacionados con el aprendizaje instrumental en las escuelas a través de convenios con ayuntamientos, institutos, escuelas de música, conservatorios y músicos profesionales. De este modo, el alumnado tiene acceso a una nueva gama de instrumentos musicales, permitiéndoles no solo su aprendizaje individual, sino también la posibilidad de formar parte de un conjunto instrumental en su centro escolar (Rickard et al., 2012).

@ Josep Gustems-Carnicer, Diego Calderón-Garrido, Mercè Navarro y Gloria Segura. The content of this article is the sole responsibility of the authors. The Revista Electrónica de LEEME and Universitat de València are not liable for any legal actions that may arise involving the article's content. Revista Electrónica de LEEME - Lista Electrónica Europea de Música en la Educación-. http://ojs.uv.es/index/php/LEEME/index ISSN: 1575-9563. Editores: Universidad de Valencia y Jesús Tejada. Visibilidad de esta revista: SCOPUS, Emerging Sources Citation Index (Clarivate), EBSCO, CINDOC (CSIC), Citefactor, COPAC, Dialnet, DICE (CSIC), DOAJ, e-revistas (CSIC), EBSCO Premier, ERIH+, Gale Cengage Learning, IN-RECS, IRESIE, LATINDEX, MIAR, OCLC Worldcat, RESH, REDIB, RILM Core Journals, SUDOC, ULRICHS. Esta revista es de acceso libre mediante licencia Creative Commons $4.0 \mathrm{CC}$ by. Política de archivado: etiqueta verde SHERPA-ROMEO. 
Quizás la clave la tenga el contexto, el conocimiento que tiene el actual profesorado sobre su uso y el interés en mantener la presencia en las aulas de un instrumento acústicomelódico, o bien optar por percusiones o recursos tecnológicos. No cabe duda de que los retos planteados son muchos y que las respuestas que se darán también lo serán. Lo importante es que guarden coherencia con el contexto escolar y persigan una educación musical de calidad en pro del desarrollo competencial del alumnado.

La formación del profesorado, por su parte, debería asegurar la adquisición de las competencias docentes mínimas necesarias para que el futuro profesorado de educación musical de los centros pueda sentirse competente en su uso y enseñanza. A la vez, dicha formación debería impulsar la implantanción de propuestas musicales innovadoras que incluyan este instrumento y a su vez, investiguen sobre la potencialidad de la experiencia musical que puede aportar a la educación del futuro. De este modo, garantizaríamos la vigencia de este instrumento en la educación musical del, ya mayor de edad, siglo XXI.

\section{Referencias}

Ben-Tovim, A. y Boyd, D. (1987). Cómo escoger el instrumento más adecuado para su hijo. Barcelona: Urano.

Bisquerra, R. (2004). Metodología de la investigación educativa. Madrid: La muralla.

Bowles, C. (1999). Actividades musicales preferidas de los estudiantes de primaria. Música y Educación, 38, 148-149.

Calderón-Garrido, D. (Coord.) (2014). Expressió musical a primària. Barcelona: Publicacions i Edicions de la Universitat de Barcelona.

Calderón-Garrido, D., Cisneros, P., García, I. y de las Heras, R. (2019). La tecnología digital en la educación musical: una revisión de la literatura científica. Revista Electrónica Complutense de Investigación en Educación Musical, 16, 4355. doi:10.5209/reciem.60768

Cateura, M. (1976). Formación Musical en la Educación Básica. Barcelona: Clivis.

Chao, R., Mato, MaD. y López, V. (2015). La formación musical del profesorado especialista en los CEIP gallegos. Revista Portuguesa de Educação, 28(2), 111-131. doi:10.21814 / rpe. 7750

Egger, J.O., Springer, D.G. y Gooding, L.F. (2013). The Effect of Behavioral Contracting on Preservice Elementary Teachers' Performance Achievement on the Soprano Recorder. Journal of Music Teacher Education, 24(2), 74-87. doi:10.1177/1057083713512318

@ Josep Gustems-Carnicer, Diego Calderón-Garrido, Mercè Navarro y Gloria Segura. The content of this article is the sole responsibility of the authors. The Revista Electrónica de LEEME and Universitat de València are not liable for any legal actions that may arise involving the article's content. Revista Electrónica de LEEME - Lista Electrónica Europea de Música en la Educación-. http://ojs.uv.es/index/php/LEEME/index ISSN: 1575-9563. Editores: Universidad de Valencia y Jesús Tejada. Visibilidad de esta revista: SCOPUS, Emerging Sources Citation Index (Clarivate), EBSCO, CINDOC (CSIC), Citefactor, COPAC, Dialnet, DICE (CSIC), DOAJ, e-revistas (CSIC), EBSCO Premier, ERIH+, Gale Cengage Learning, IN-RECS, IRESIE, LATINDEX, MIAR, OCLC Worldcat, RESH, REDIB, RILM Core Journals, SUDOC, ULRICHS. Esta revista es de acceso libre mediante licencia Creative Commons $4.0 \mathrm{CC}$ by. Política de archivado: etiqueta verde SHERPA-ROMEO. 
Escalas, R. (1972). Flauta dulce soprano (y tenor). Técnica básica elemental. Barcelona: Quiroga.

Ferrer, R. (2009). El canto coral y las orquestas infantiles, una educación en valores. Eufonía, Didáctica de la Música, 45, 30-38.

Fitch, W.T. (2005). The Evolution of Music in Comparative Perspective. Annals of New York Academy of Sciences, 1060, 1-21. doi:10.1196/annals.1360.004

Galofré, F. (1980). La Flauta Dolça. Mètode d'Iniciació per a Infants. Barcelona: Dossiers Rosa Sensat.

García, E., del Olmo, M.J. y Gutiérrez-Rivas, E. (2014). Educación musical y desarrollo cognitivo asociado. Música y Educación, 97, 28-41.

Gustems, J. (2004). La Flauta dulce en los estudios universitarios de "Mestre en Educació Musical" en Catalunya: revisión y adecuación de contenidos [Tesis doctoral, Universitat de Barcelona]. Recuperado de: http://www.tdx.cesca.es/TDX-0716104$\underline{104533}$

Gustems, J. y Cunill, G. (2007). Recursos electrònics $i$ audiovisuals per l'ensenyament $i$ aprenentatge de la flauta dolça. Barcelona: Recercat. Recuperado de: http://hdl.handle.net/2445/11540

Gustems-Carnicer, J., y Calderón-Garrido, D. (2016). Proyectos musicales, ciudadanía y desarrollo humano: una mirada desde la psicología positiva. Cuadernos de Música, Artes Visuales y Artes Escénicas, 11 (2), 251-272. doi:10.11144/Javeriana.mavae112.pmcd

Hunt, E. (1978). La Flûte à Bec et sa Musique. París: Zurfluh.

Huillipan Rain, J. y Ángel-Alvarado, R. (2020). Arreglos musicales en el aula: Factores pedagógicos en la Educación Primaria. Revista Electrónica de LEEME, 45, 53-68. doi:10.7203/LEEME.45.16527

Izquierdo, J., Jiménez, R. y Montserrat, R.M. (1996). Peix de Sabó 1, Flauta de Bec, Cicle Mitjà. Barcelona: Barcanova.

Jambrina, M.E. (2007). La flauta dulce en el área de expresión artística en la educación primaria: Comunidad autónoma de Extremadura. Realidad, implicaciones y propuestas para el profesorado. Badajoz: Universidad de Extremadura.

@ Josep Gustems-Carnicer, Diego Calderón-Garrido, Mercè Navarro y Gloria Segura. The content of this article is the sole responsibility of the authors. The Revista Electrónica de LEEME and Universitat de València are not liable for any legal actions that may arise involving the article's content. Revista Electrónica de LEEME - Lista Electrónica Europea de Música en la Educación-. http://ojs.uv.es/index/php/LEEME/index ISSN: 1575-9563. Editores: Universidad de Valencia y Jesús Tejada. Visibilidad de esta revista: SCOPUS, Emerging Sources Citation Index (Clarivate), EBSCO, CINDOC (CSIC), Citefactor, COPAC, Dialnet, DICE (CSIC), DOAJ, e-revistas (CSIC), EBSCO Premier, ERIH+, Gale Cengage Learning, IN-RECS, IRESIE, LATINDEX, MIAR, OCLC Worldcat, RESH, REDIB, RILM Core Journals, SUDOC, ULRICHS. Esta revista es de acceso libre mediante licencia Creative Commons 4.0 CC by. Política de archivado: etiqueta verde SHERPA-ROMEO. 
Kang, S. (2018). Motivation and Preference for Acoustic or Tablet-Based Musical Instruments: Comparing Guitars and Gayageums. Journal of Research in Music Education, 66(3), 278-294. doi:10.1177/0022429418785379

Kokotsaki, D. y Hallam, S. (2007). Higher education music students' perceptions of the benefits of participative music making. Music Education Research, 9(1), 93-109. doi:10.1080/14613800601127577

Lafarga, M. y Sanz, P. (2014). Flautas Prehistóricas. Música y Educación, 97, 54.

Lander, N.S. (1996-2020). Recorder Home Page: History. Recuperado de: https://www.recorderhomepage.net/history/

López de Arenosa, E. (2016). Desde la distancia. Una mirada a los conservatorios superiores de música españoles. En A. Vernia (Coord.). Las enseñanzas superiores de música en el territorio español (pp.69-91). Logroño: Siníndice.

Lubarsky, E.M. (2017). Reviving early music: metaphors and modalities of life and living in historically informed performance. Rochester: University of Rochester.

Martínez, R., Castellanos, M.A. y Chacón, J.C. (2014). Análisis de datos en Psicología y Ciencias de la Salud. Volumen II. Inferencia estadística. Madrid: EOS Universitaria.

Montoya, J.C. (2017). El recorrido de las metodologías de principios del siglo XX en la enseñanza de la música en España: aproximación bibliográfica e interpretativa. Anuario Musical, 72, 219-232. doi:10.3989/anuariomusical.2017.72.10

Orff, C. y Keetman, G. (1950). Musik für Kinder. Vol 1. Im Fünftonraum. Mainz: Mainz Schott.

Oriol, N. (2004). Metodología cuantitativa y cualitativa en la investigación sobre la formación inicial del profesorado de educación musical para primaria. Aplicación a la formación instrumental. Revista Electrónica Complutense de Investigación en Educación Musical - RECIEM, 1, 1-63. Recuperado de: https://core.ac.uk/download/pdf/25879609.pdf

Paquier, M., Hendrickx, E. y Jeannin, R. (2016). Effect of wood on the sound of oboe as simulated by the chanter of a 16-inch French bagpipe. Applied Acoustics, 103, 47-53. doi:10.1016/j.apacoust.2015.10.008

Rickard, N.S., Appelman, P., James, R., Murphy, F., Gill, A. y Bambrick, C. (2012). Orchestrating life skills: The effect of increased school-based music classes on children's social competence and self-esteem. International Journal of Music Education, 31(3), 292-309. doi:10.1177/0255761411434824

@Josep Gustems-Carnicer, Diego Calderón-Garrido, Mercè Navarro y Gloria Segura. The content of this article is the sole responsibility of the authors. The Revista Electrónica de LEEME and Universitat de València are not liable for any legal actions that may arise involving the article's content. Revista Electrónica de LEEME - Lista Electrónica Europea de Música en la Educación-. http://ojs.uv.es/index/php/LEEME/index ISSN: 1575-9563. Editores: Universidad de Valencia y Jesús Tejada. Visibilidad de esta revista: SCOPUS, Emerging Sources Citation Index (Clarivate), EBSCO, CINDOC (CSIC), Citefactor, COPAC, Dialnet, DICE (CSIC), DOAJ, e-revistas (CSIC), EBSCO Premier, ERIH+, Gale Cengage Learning, IN-RECS, IRESIE, LATINDEX, MIAR, OCLC Worldcat, RESH, REDIB, RILM Core Journals, SUDOC, ULRICHS. Esta revista es de acceso libre mediante licencia Creative Commons $4.0 \mathrm{CC}$ by. Política de archivado: etiqueta verde SHERPA-ROMEO. 
Robert, C. (1998). XXème Siècle et Flûte à Bec. Sa redécouverte en France. Bourg-la-Reine: Zurfluh.

Rodríguez, J.R. y Álvarez, R.M.V. (2017). The music materials in early childhood education: A descriptive study in Galicia (Spain). International Journal of Music Education, 35(2), 139-153. doi:10.1177/0255761415619423

Roudabush, K. (2017). The flute in transition: a comparison of extant flutes from circa 1650 to 1715 [Tesis doctoral, Indiana University]. Recuperado de: https://cutt.ly/FrMoW9h

Thomson, J.M. (1995). The Cambridge Companion to the Recorder. Cambridge: Cambridge University Press.

Tripiana, S. (2016). Importancia de las estrategias de práctica instrumental en la educación musical superior. Revista Electrónica Complutense de Investigación en Educación Musical, 13, 64-88. doi:10.5209/RECIEM.51606

Wrape, E.R., Dittloff, A.L. y Callahan, J.L. (2016). Gender and musical instrument stereotypes in middle school children: Have trends changed? Update: Applications of Research in Music Education, 34(3), 40-47. doi:10.1177/8755123314564255

Young, V., Burwell, K. y Pickup, D. (2003). Areas of Study and Teaching Strategies in Instrumental Teaching: a case study research project. Music Education Research, 5(2), 139-154. doi:10.1080/1461380032000085522

@ Josep Gustems-Carnicer, Diego Calderón-Garrido, Mercè Navarro y Gloria Segura. The content of this article is the sole responsibility of the authors. The Revista Electrónica de LEEME and Universitat de València are not liable for any legal actions that may arise involving the article's content. Revista Electrónica de LEEME - Lista Electrónica Europea de Música en la Educación-. http://ojs.uv.es/index/php/LEEME/index ISSN: 1575-9563. Editores: Universidad de Valencia y Jesús Tejada. Visibilidad de esta revista: SCOPUS, Emerging Sources Citation Index (Clarivate), EBSCO, CINDOC (CSIC), Citefactor, COPAC, Dialnet, DICE (CSIC), DOAJ, e-revistas (CSIC), EBSCO Premier, ERIH+, Gale Cengage Learning, IN-RECS, IRESIE, LATINDEX, MIAR, OCLC Worldcat, RESH, REDIB, RILM Core Journals, SUDOC, ULRICHS. Esta revista es de acceso libre mediante licencia Creative Commons $4.0 \mathrm{CC}$ by. Política de archivado: etiqueta verde SHERPA-ROMEO. 